\title{
A Survey on Software Reuse Processes
}

\author{
Eduardo Santana de Almeida ${ }^{1}$, Alexandre Alvaro ${ }^{1}$, Daniel Lucrédio ${ }^{2}$, Vinicius Cardoso Garcia ${ }^{2}$, \\ Silvio Romero de Lemos Meira ${ }^{1}$ \\ ${ }^{1}$ Federal University of Pernambuco and C.E.S.A.R. - Recife Center for Advanced Studies and \\ Systems, ${ }^{2}$ Federal University of São Carlos, Brazil \\ \{esa2,aa2,srlm\}@cin.ufpe.br, \{lucredio,vinicius\}@dc.ufscar.br
}

\begin{abstract}
Software reuse processes have been under continuous attention in the software engineering and software reuse research communities during past years. Although several processes have been investigated to develop reusable software, there are not available studies that compare them. In this way, this paper presents a detailed survey on software reuse processes.
\end{abstract}

\section{Introduction}

Software reuse is a critical aspect for companies interested in the improvement of software development quality and productivity [1]. Quality could be improved by reusing all forms of proven experience, including products and processes, as well as quality and productivity models. Productivity could increase by reusing existing experience, rather than creating everything from the beginning [2].

Through the years, several research works, including company reports $[3,4,5,6,7]$, informal research [8] and empirical studies $[9,10,11]$ have shown that an effective way to obtain the software reuse benefits is to adopt a reuse process. However, the existing reuse processes present crucial problems such as gaps in important activities like development for and with reuse, and putting more emphasis on some specific activities (analysis, design and implementation). Even today, with the ideas of software product lines, there is still no clear consensus about the activities (inputs, outputs, artifacts) and the requirements that an effective reuse process must have.

In this context, we agree with Bayer et al. when they say [12] (pp. 122): "Existing methods have been either not flexible enough to meet the needs of various industrial situations, or they have been too vague, not applicable without strong additional interpretation and support. A flexible method that can be customized to support various enterprise situations with enough guidance and support is needed".

Under this motivation, this paper presents a detailed survey based on eleven software reuse processes representing the state-of-the-art in the area. We believe that this survey can contribute with the development in the area, as well as with the definition of new reuse processes.

\section{Software Reuse Processes: A Survey}

The argument for defining processes for specific software development tasks should be a familiar one. A well-defined process can be observed and measured, and thus improved. Moreover, the adoption of processes also allows the dissemination of effective work practices to occur more quickly than the building up of personal experience. An emphasis on process helps software development to become more like engineering, with predictable time and effort constraints, and less like art [13].

Besides the issues related to non-technical aspects, a software reuse process must also describe two essential activities: the development for reuse and the development with reuse. In the literature, several research works that study efficient ways to develop reusable software can be found. These works focus on two directions: domain engineering and, currently, product lines, as can be seen in the next sections.

\subsection{Domain Engineering Processes}

Domain engineering is the activity of collecting, organizing, and storing past experience in building systems or parts of systems in a particular domain in the form of reusable assets, as well as providing an adequate means for reusing these assets when building new systems [14].

Among the works of the early 80 's and 90 's, such as $[15,16,17,18,19,20]$, a special focus is placed on the domain engineering processes to develop reusable software.

An example of this work may be seen in [15]. In this work, Neighbors proposed the first domain engineering approach, as well as a prototype - Draco - based on transformation technology. The main ideas introduced by Draco include: Domain Analysis, Domain-Specific Languages, and Components as sets of transformations.

Draco supports the organization of software construction knowledge into a number of related domains. Each Draco domain encapsulates the needs and 
requirements and different implementations of a collection of similar systems.

Neighbors' work provided an important contribution for the domain engineering field, presenting concepts such as generative programming, transformation systems and components. Nevertheless, his approach is very difficult to apply in the industrial environment due the complexity to perform activities such as writing transformations and using the Draco machine. Even with some advances related to his work [21], many of these problems still remain unsolved. Thus, even presenting a potential possibility to develop reusable software, the software reuse community and the industry needed a major guidance to achieve this concept in an effective way.

In this context, in 1992 [16], the Software Technology for Adaptable, Reliable Systems (STARS) developed the Conceptual Framework for Reuse Processes (CFRP) as a vehicle for understanding and applying the STARS domain-specific reuse-based software engineering paradigm. The CFRP established a framework for considering reuse-related software engineering processes, how they interrelate, and how they can be integrated with each other and with non-reuse-related processes to form reuse-oriented life-cycle process models that are tailorable to organization needs.

However, the CFRP by itself was a very generic framework, and organizations found out that the CFRP was too generic to serve as the sole basis for developing a tailored domain-specific reuse-based software engineering life cycle model. Thus, the ROSE Process Model (ROSE PM) [16] was developed by the Paramax STARS team specifically to bridge the gap between the high-level, generic framework and the detailed, prescriptive methods.

ROSE PM is divided into four iterative submodels: Organization Management, Domain Engineering, Asset Management, and Application Engineering. Organization Management consists of the highest-level organizational Plan, Enact, and Learn activities. In the ROSE model, the project (Domain Engineering, Asset Management, and Application Engineering) activities are hierarchically placed within the Enact portion of Organization Management. The goal of the ROSE Domain Engineering project submodel is to capture, organize and represent knowledge about a domain and produce reusable assets that can be applied to produce a family of systems encompassing that domain. In the Asset Management submodel the goal is to acquire, evaluate, and organize assets produced by domain engineering, and make those assets available as a managed collection that serves as mediation or brokering mechanisms between asset creators and asset utilizers. Finally, in the Application Engineering the goal is to develop, reengineer, maintain, or evolve software systems, making use of assets created in domain engineering.

The key objective of the ROSE model is to integrate software maintenance and reengineering in the context of domain-specific reuse to produce a general reuse-oriented approach to software evolution. However, the main problem with ROSE is that the process model presents the activities in a generic way without specifying, in details, how they should be performed. For example, in Domain Engineering, there is an activity defined as Develop Software Components, but how to perform it is not defined. The same problem happens in other activities such as Develop Software Architecture and Develop Applications Generators.

Four years after the beginning of the efforts in the STARS project, Mark Simos [17] and his group developed the Organization Domain Modeling (ODM) method. Their motivation was that there was a gap in the domain engineering methods and processes available to reuse practitioners. Simos' thesis was that because work in domain analysis for software reuse emerged out of the software engineering research community, many of the methods were developed by technologists. Moreover, Simos highlighted that people trying to launch small-scale domain engineering projects have to negotiate technical and non-technical issues in planning, managing, and transitioning their work. Yet they typically do not have the support of an already existing reuse infrastructure within the organization.

In fact, to motivate organizations to adopt reuse practices on a more global scale may well depend on successful results from initial pilot projects. Thus, the ODM method was an attempt to meet this need and establish a foundation for evolving initial pilot projects into reuse programs.

The ODM process consists of three main phases: Plan Domain, Model Domain, and Engineer Asset Base. The purpose of the Plan Domain phase is to set objectives and select and scope a domain for the project in alignment with overall organizational needs. In Model Domain phase the goal is to produce a domain model for the selected domain, based on the domain definition, produced in the previous phase. Finally, in Engineer Asset Base, the purpose is to scope, architect, and implement an asset base that supports a subset of the total range of variability encompassed by the domain model. Plan Domain and Model Domain correspond to domain analysis. Engineer Asset Base encompasses both domain design and implementation.

Although ODM encompasses the steps of domain engineering, it does not present specific details on how to perform many of its activities, as happens in ROSE. However, this limitation is clearly described in the ODM guidebook. According to Simos et al. the method provides a "general, high-level guidance in tailoring the 
method for application within a particular project or organization" (pp. 01). In this way, critical activities are not properly guided, which can contribute to possible problems for organizations using the method.

Until Simos' work, the research involving software reuse processes were too general, without presenting concrete techniques to perform tasks such as architecture and component modeling and implementation. Moreover, it was difficult to develop reusable object-oriented software using these processes.

In this context, three software development experts Jacobson, Griss and Jonsson - created the Reuse-driven Software Engineering Business (RSEB) [18]. RSEB is a use-case driven systematic reuse process based on UML notation. The method was designed to facilitate both the development of reusable object-oriented software and software reuse. Similar to the Unified Process [22], RSEB is also iterative and use-case centric.

Key ideas in RSEB are: the explicit focus on modeling variability and to maintain traceability links connecting representation of variability throughout all models, i.e., variability present in use cases can be traced to variability in the analysis, design, and implementation object models.

RSEB has separated processes for Domain Engineering and Application Engineering. Domain Engineering in RSEB consists of two processes: Application Family Engineering, which develops and maintains the overall layered system architecture, and Component System Engineering, which develops component systems for the different parts of the application system with a focus on building and packaging robust, extendible, and flexible components.

In RSEB, Application Engineering is composed of steps to: Capture requirements, Perform robustness analysis, Design the application system, Implement the application system, Test the application system and Package the application system.

Despite the RSEB focus on variability, the process components of Application Family Engineering and Component System Engineering do not include essential domain analysis techniques such as domain scoping and feature modeling. Moreover, the method does not describe a systematic way to perform the asset development as proposed. Another shortcoming of RSEB is the lack of feature models. In RSEB, variability is expressed at the highest level in the form of variation points, which are then implemented in other models using variability mechanisms.

Based on the limitations presented with the RSEB utilization, Griss et al. developed FeatuRSEB [19], which is a result of integrating FODAcom, an object-oriented adaptation of FODA [23] for the telecom domain, into RSEB, through a cooperation between Hewlett-Packard and Intecs Sistemi.
FeatuRSEB extends RSEB in two points: i. the activity that corresponds to Domain Analysis was extended with steps to perform domain scoping (briefly) and feature modeling and ii. feature models are used as the main representation of commonality, variability, and dependencies.

Although FeatuRSEB had presented important considerations related to domain analysis, such as the process of extracting functional features from the domain use case model, for example, the limitations discussed in FeatuRSEB were not solved.

Concurrently with Griss et al.'s work, a respected researcher in the domain analysis area, Kyo Kang, in conjunction with his colleagues, presented the thesis that there were many attempts to support software reuse, but most of these efforts have focused on two directions: exploratory research to understand issues in domain specific software architectures, component integration and application generation mechanisms; and theoretical research on software architecture and architecture specification languages, development of reusable patterns, and design recovery from existing code. Kang et al. considered that there were few efforts to develop systematic methods for discovering commonality and using this information to engineer software for reuse. This was their motivation to develop the Feature-Oriented Reuse Method (FORM) [20], an extension of their previous work [23].

FORM is a systematic method that focuses on capturing commonalities and differences of applications in a domain in terms of features and using the analysis results to develop domain architectures and components. In FORM, the use of features is motivated by the fact that customers and engineers often speak of product characteristics in terms of features the product has and/or delivers. They communicate requirements or functions in terms of features and, to them, features are distinctively identifiable functional abstractions that must be implemented, tested, delivered, and maintained.

The FORM method consists of two major engineering processes: domain engineering and application engineering. The domain engineering process consists of activities for analyzing systems in a domain and creating reference architectures and reusable components based on the analysis results. The application engineering process consists of activities for developing applications using the artifacts created in the domain engineering.

The core of FORM lies in the analysis of domain features and use of these features to develop reusable domain artifacts. The domain architecture, which is used as a reference model for creating architectures for different systems, is defined in terms of a set of models, each one representing the architecture at a different level of abstraction. Nevertheless, aspects such as specification, 
design, implementation and packaging of the components are little explored.

After the domain development, the application engineering process is performed. Once again, the emphasis is in the analysis phase, with the use of the developed features. However, few directions are defined to select the architecture model and develop the applications using the existing components.

\subsection{Product Line Processes}

Until 1998, the software reuse processes were only related to domain engineering issues. However, in this same period, a new trend started be explored: the software product line area [24]. Software product lines began to be seen as one of the most promising advances for efficient software development. However, until the late 90 's there were few guidelines or methodologies available to develop and deploy product lines beyond existing domain engineering approaches.

On the other hand, domain engineering approaches have not proved to be as effective as expected. According to Bayer et al. [12], there are basically three reasons for this: misguided scoping of application area, lack of operational guidance, and overstressed focus on organizational issues. Moreover, domains have proved to be difficult to scope and engineer from an enterprise point of view because a domain captures many extraneous elements that are of no interest to an enterprise. Hence, the domain view provides little economic basis for scoping decisions. Instead, enterprises focus on particular products (existing, under development, and anticipated). This difference in focus is essential for practically supporting the product-driven needs of enterprises.

In this context, based on the mentioned domain engineering's limitations and lack of product-lines approaches, Bayer et al. proposed the Product Line Software Engineering (PuLSE) methodology. The methodology was developed with the proposal of enabling the conception and deployment of software product lines within a large variety of enterprise contexts. One important feature of PuLSE is that it is the result of a bottom-up effort: the methodology captures and leverages the results (the lessons learned) from technology transfer activities with industrial customers.

PuLSE is composed of three main elements: the deployment phases, the technical components, and the support components. The deployment phases are a set of stages that describe activities for initialization, infrastructure construction, infrastructure usage, and evolution and management of product lines. The technical components provide the technical know-how that is need to operationalize the product line development. At the end, the support components are packages of information, or guidelines, which enable a better adaptation, evolution, and deployment of the product line.
PuLSE methodology presents an initial direction to develop software product lines. However, some points are not well discussed. For example, the component PuLSEDSSA supports the definition of a domain-specific software architecture, which covers current and future application of the product line. Nevertheless, aspects such as specification, design, and implementation of the architecture's components are not presented. Bayer et al. consider it an advantage, because PuLSE-DSSA dos not require a specific design methodology nor a specific Architecture Description Language (ADL). We do not agree with this vision, because, in our opinion, the lack of details is the big problem related to software reuse processes. The same problem can be seen in the Usage phase, in which are specified, derived and validated product lines members without explicit details of how to make it.

According to Atkinson et al. [25], PuLSE has been applied successfully in various contexts with different purposes. Among the benefits, it proved to be helpful for introducing sound documentation and development techniques into existing development practices. However, in circumstances where there were no pre-existing processes or well-defined products, the introduction of PuLSE turned out to be problematic. In such cases, the customization of PuLSE was actually more concerned with the introduction of basic software engineering processes than with the adaptation of the product line ideas in relation to existing processes.

From this perspective, the KobrA approach was proposed [25]. The approach represents a synthesis of several advanced software engineering technologies, including product line, component-based development, frameworks, and process modeling.

KobrA has two main activities: initially, framework engineering creates and maintains a generic framework that embodies all product variant that make up the family, including information about their common and disjoint features. Next, application engineering uses the framework that was built during framework engineering to construct specific applications in the domain covered by the framework. In KobrA, a framework is the static representation of a set of Komponents (KobrA component) organized in the form of a tree. Each Komponent is described at two levels of abstraction: a specification, which defines the Komponent's externally visible properties and behavior, and a realization, which describes how the Komponent fulfills the contracts with other Komponents.

Even supporting the development for and with reuse, KobrA presents some drawbacks. For example, during the framework engineering activity, it does not present guidelines to perform tasks such as domain analysis and domain design. This question is a little strange, because 
PuLSE clearly supported activities for planning and scoping.

In the same time, another important and different point of view related to product line processes was proposed in [26]. According to America et al., software reuse processes are strongly related to non-technical aspects such as the business and the organizational constraints. America et al. mention the case of the Philips company, where several product families are developed, ranging from consumer electronics to professional systems. For each of these families a specific family engineering method is necessary, because the business and organization constraints differ. Based on this motivation, the Component-Oriented Platform Architecting Method (CoPAM) Family for Product Family Engineering was proposed. Thus, for each product family, the CoPAM approach develops a specific family engineering method from the method family in a step called method engineering.

According to America et al., the reason why they did not considerate an alternative approach that would organize the different product families into one larger family or population was that a large population only makes sense if the products have enough in common to make it profitable to develop them as a population and the organization is able to coordinate the marketing, development, and other aspects of a large population.

The CoPAM processes are based on several approaches described in the literature, such as PULSE, RSEB, RUP, and on authors' experience in industry. The processes have two main subprocesses: platform engineering and product engineering. Platform engineering process develops a platform, which consists of a number of reusable components. A product engineering process develops products using these platform components, adding new components where necessary. Both of these receive guidance from and provide feedback to the family engineering process.

In CoPAM, there is a single family engineering process per product family, but there can be more than one platform or product engineering subprocesses. The family engineering process has seven steps leading to the detailed component architecture for the family. The steps range from informal domain analysis to family architecting and support. However, details about how to achieve specific tasks, such as, for example, domain analysis or family architecting, are not presented.

Until 2000, the software reuse processes were related to the software engineering domain. However, a collaborative project between industry and academy, starting in October 2000 and finishing in September 2002, applied the reuse concepts on embedded systems domain [27]. The Pervasive Component Systems (PECOS) project goal was to enable component-based development for embedded systems. Its motivation was that embedded systems are dificult to maintain, upgrade and customize, and they are almost impossible to port to other platforms. In this context, reuse concepts, mainly, component-based development could present relevant improvements.

The PECOS process focuses on two central issues: the first question is how to enable the development of families of PECOS devices. The second issue concerns the composition of a PECOS field device from an asset prefabricated components and how these components have to be coupled, not only on the functional, but also on the non-functional level.

The development of a PECOS component is performed during application composition when the need for a component is identified and this component cannot be provided from the component repository. The component development is achived in five tasks: Requirements elicitation, Interface specification, Implementation, Test \& Profiling, and Documentation \& Release. An interesting task of this cycle is Profiling, which collects detailed non-functional information about the components. The application development is composed of activities to identify, query, select and compose applications reusing components.

Even presenting an important contribution to the embedded systems domain, the PECOS process presents some gaps and drawbacks. For example, in component development, it does not discuss how the requirements elicitation is performed, how the contracts among the components are specified, and how the components are identified. Moreover, the components are not developed in tasks such as domain analysis. In PECOS, the components are contructed in an isolated way, without this view.

At the end, the last process related to product line is seen in [28]. In this work, Kang et al. present an evolution of their previous work, FORM [20]. FORM was extended in order to support the development of software product lines.

FORM's product line consists of two processes: asset development and product development. Asset development consists of analyzing a product line (such as marketing and product plan development, feature modeling, and requirements analysis) and developing architectures and reusable components based on analysis results.

Product development includes analyzing requirements, selecting features, selecting and adopting an architecture, adapting components and, finally, generating code for the product.

The major contribution of this work, when compared to FORM, is the concern with business aspects such as marketing and product plan (MPP). The MPP identifies the information to gather during the marketing and business analysis. It includes a market analysis, a 
marketing strategy, product features, and product feature delivery methods.

On the other hand, the process presents lack of details in specific activities, such as conceptual architecture design and architecture refinement. In these activities, there are no information on how to identify the components and specify dependencies among them. Moreover, the process of product development and its activities are not discussed.

\section{Related Works}

In [29], Arango presented a comparative study of domain analysis methods. In [30] and [31], Boertin et al. and Stojanovic et al. performed similar analysis involving component-based development methods, including Catalysis, Rational Unified Process (RUP), and Select Perspective, based on their maturity and fitness-for-use in the context of e-business and software engineering. Finally in [32], Matinlassi discusses a study for product line architecture evaluation based on five methods for product lines including FORM, CoPAM and KobrA.

Our study is different from the cited because it is focused on software reuse processes including all life cycle, not only specific steps such as domain analysis, architecture design or component-based development.

\section{Summary}

Adopting a software reuse process is an effective way for organizations to obtain the benefits related to software reuse, such as quality improvements and time-to-market reduction. However, the choice of a reuse process is not a trivial task, even considering the division between domain engineering and product lines.

In this paper, we surveyed eleven software reuse processes corresponding to the state-of-the-art in the area. This survey may serve as a guide to aid organizations in the adoption of a reuse process, or as a basis for a formation of a reuse process. Currently, we are working on the software reuse process formalization, in the domain analysis step, defining methods for domain scoping and feature modeling. This project is part of the Reuse in Software Engineering (RiSE) project [33]. RiSE's goal is to develop a robust framework for software reuse, in conjunction with the industry, involving processes, methods, environment and tools.

\section{References}

[1] C.W. Krueger, Software Reuse, ACM Computing Surveys, Vol. 24, No. 02, June, 1992, pp. 131-183.

[2] V.R. Basili, L.C. Briand, W.L. Melo, How Reuse Influences Productivity in Object-Oriented Systems, Communications of the ACM, Vol. 39, No. 10, October, 1996, pp. 104-116.

[3] A. Endres, Lessons Learned in an Industrial Software Lab, IEEE Software, Vol. 10, No. 05, September, 1993, pp. 58-61.

[4] D. Bauer, A Reusable Parts Center, IBM Systems Journal, Vol. 32, No. 04, 1993, pp. 620-624.

[5] M.L. Griss, Software Reuse Experience at Hewlett-Packard, Proceedings of the 16th ICSE, 1994

[6] M.L. Griss, Making Software Reuse Work at Hewlett-Packard, IEEE Software, Vol. 12, No. 01, January, 1995, pp. 105-107.
[7] R. Joos, Software Reuse at Motorola, IEEE Software, Vol. 11, No. 05, September, 1994, pp. 42-47.

[8] W.B. Frakes, S. Isoda, Success Factors of Systematic Software Reuse, IEEE Software, Vol. 12, No. 01, January, 1995, pp. 14-19.

[9] D.C. Rine, Success Factors for software reuse that are applicable across Domains and businesses, ACM Symposium on Applied Computing, 1997, pp. 182-186.

[10] M. Morisio, M. Ezran, C. Tully, Success and Failure Factors in Software Reuse, IEEE Transactions on Software Engineering, Vol. 28, No. 04, April, 2002, pp. 340-357.

[11] M.A. Rothenberger, K.J. Dooley, U.R. Kulkarni, N. Nada, Strategies for Software Reuse: A Principal Component Analysis of Reuse Practices, IEEE Transactions on Software Engineering, Vol. 29, No. 09, September, 2003, pp. 825-837.

[12] J. Bayer, O. Flege, P. Knauber, R. Laqua, D. Muthig, K. Schmid, T. Widen, J. DeBaud, PuLSE: A Methodology to Develop Software Product Lines, Symposium on Software Reusability (SSR), 1999, pp. 122-131.

[13] D. Rombach, Fraunhofer: The German Model for Applied Research and Technology Transfer, ICSE, 2000, pp. 25-34.

[14] K. Czarnecki, U. W. Eisenecker, Generative Programming: Methods, Tools, and Applications, Addison-Wesley, 2000, p. 832.

[15] J.M. Neighbors, Software Construction Using Components, PhD Thesis, University of California, USA, 1980, p. 75.

[16] Software Technology for Adaptable, Reliable Systems (STARS), The Reuse-Oriented Software Evolution (ROSE) Process Model, Technical Report, July, 1993, p. 143.

[17] M. Simos, D. Creps, C. Klingler, L. Levine, D. Allemang, Organization Domain Modeling (ODM) Guidebook Version 2.0, Technical Report, June, 1996, p. 509.

[18] I. Jacobson, M.L. Griss, P. Jonsson, Reuse-driven Software Engineering Business (RSEB), Addison-Wesley, 1997, p. 497.

[19] M.L. Griss, J. Favaro, M. d' Alessandro, Integrating Feature Modeling with the RSEB, ICSR, 1998, pp. 76-85.

[20] K.C. Kang, S. Kim, J. Lee, K. Kim, E. Shin, M. Huh, FORM: A Feature-Oriented Reuse Method with domain-specific reference architectures, Annals of Software Engineering Notes, 1998, pp. 143-168. [21] J.C.S.P. Leite, Draco-Puc: A Technology Assembly for Domain Oriented Software Development, ICSR, 1994, pp. 94-100.

[22] I. Jacobson, G. Booch, J. Rumbaugh, The Unified Software Development Process, Addison-Wesley, February, 1999, p. 463.

[23] K.C. Kang, S.G. Cohen, J.A. Hess, W.E. Novak, A.S. Peterson, Feature-Oriented Domain Analysis (FODA) Feasibility Study, Technical Report CMU/SEI-90-TR-21, 1990.

[24] P. Clements, L. M. Northrop, Software Product Lines: Practices and Patterns, Addison Wesley, August, 2001, p. 608.

[25] C. Atkinson, J. Bayer, D. Muthig, Component-Based Product Line Development: The KobrA Approach, The 1st SPLC, 2000, p.19.

[26] P. America, H. Obbink, R. V. Ommering, F. V. D. Linden, CoPAM: A Component-Oriented Platform Architecting Method Family for Product Family Engineering, The 1st SPLC, 2000, p.15.

[27] M. Winter, C. Zeidler, C. Stich, The PECOS Software Process, Workshop on CBD, in 7th ICSR, 2002, p.07.

[28] K. C. Kang, J. Lee, P. Donohoe, Feature-Oriented Product Line Engineering, IEEE Software, July/August, 2002, pp.58-65.

[29] G. Arango, Domain Analysis Methods, Software Reusability, W. Schaefer, R. Prieto-Diaz, M. Matsumoto (editors), 1994, pp. 17-49.

[30] N. Boertin, M. Steen, H. Jonkers, Evaluation of Component-Based Development Methods, 6th Int. Workshop on Evaluation of Modeling Methods in Systems Analysis and Design, in Tte 13th CAiSE, 2001.

[31] A. Stojanovic, A. Dahanayake, H. Sol, A Methodology Framework for Component-Based System Development Support, 6th Int. Workshop on Evaluation of Modeling Methods in Systems Analysis and Design, in Tte 13th CAiSE, 2001

[32] M. Matinlassi, Comparison of Software Product Line Architecture Design Methods: COPA, FAST, FORM, KobrA and QUADA, Proceedings of the $26^{\text {th }}$ ICSE, 2004, pp. 127-136.

[33] E. S. Almeida, A. Alvaro, D. Lucrédio, V.C. Garcia, S.R.L. Meira, RiSE Project: Towards a Robust Framework for Software Reuse, IEEE International Conference on Information Reuse and Integration (IRI), 2004, pp. 48-53. 